\title{
Impact on ovarian reserve after minimally invasive single-port laparoscopic ovarian cystectomy in patients with benign ovarian cysts: A systematic review and meta-analysis
}

\author{
Eleni Tsiampa ${ }^{1}$, Eleftherios Spartalis ${ }^{1}$, Gerasimos Tsourouflis ${ }^{1}$, Dimitrios Dimitroulis ${ }^{1}$, and \\ Nikolaos Nikiteas ${ }^{1}$ \\ ${ }^{1}$ Affiliation not available
}

January 17, 2021

\begin{abstract}
Background/Aim: The purpose of this article is to review the published literature on single-port laparoscopic (SPL) ovarian cystectomy and to assess whether the reduced port number affects the ovarian reserve in comparison with the conventional multiport laparoscopic (MPL) ovarian cystectomy. Materials and methods: Serum anti-Müllerian hormone (AMH) had been proposed as the most accurate marker of ovarian reserve. A review of the current literature was performed based on the preoperative and postoperative AMH after SPL and MPL ovarian cystectomy in adult patients with benign ovarian cysts. Results: Ovarian cystectomy causes a non-statistically significant reduction in AMH levels four weeks postoperatively in the SPL group compared to MPL group[MD=0.11, 95\% CI $(-0.01,0.24), \mathrm{p}=0.07]$. Operative time was significantly longer and blood loss was significantly higher in SPL group. No difference was found to major or overall postoperative complications between the two groups Conclusion: SPL cystectomy recommended as a safe surgical choice for patients who want to preserve their fertility.
\end{abstract}

\section{Introduction}

Benign ovarian cysts are very common in women of reproductive age and often require surgical excision, particularly when they cause symptoms or infertility ${ }^{1}$. However, there has been a growing concern over the possible damaging effect of this procedure on ovarian reserve ${ }^{2,3}$. The continuous development of technology and minimally invasive techniques during the recent years has established laparoscopic ovarian cystectomy as the first-line treatment for benign ovarian tumors, such as symptomatic endometriomas, dermoid cysts or serous cystadenomas ${ }^{4}$. Due to favourable surgical outcomes, fewer complications, less pain, and improved cosmetic results laparoscopic cystectomy has almost replaced open surgery ${ }^{5}$. Nevertheless, several studies have indicated that laparoscopic ovarian cystectomy is associated with a decreased ovarian reserve and for this reason there has been much debate about the most suitable laparoscopic approach that causes the less damage in ovarian reserve after surgery ${ }^{6-9}$.

A recent advancement in the field of minimally invasive gynecology is transumbilical single-port laparoscopy (SPL). This minimally invasive approach to surgery requires only one entry point, typically in the umbilical region. In particular, the use of SPL in adnexal disease as well as in endometriomas excision has been proposed ${ }^{10}$. There have been clinical reports that show that a limited operative space in SPL causes unstable camera motion and interference between instruments which may increase the difficulty and risk of surgery ${ }^{11-12}$. Yoon et al., Huang et al. and Wang et al. found that the ovarian reserve did not statistically differ between single-port laparoscopic cystectomy (SPL) and conventional multiport laparoscopic cystectomy $(\mathrm{MPL})^{13-15}$. However, Angioni et al concluded that the mean anti-Müllerian hormone (AMH) concentration -an ovarian reserve indicator- significantly decreased in the SPL group at four to six-week and three-month follow-up periods compared with that in the MPL group ${ }^{16}$. 
Among other traditional biologic indicators AMH is considered to be the most accurate biomarker of ovarian reserve. AMH reflects the decreasing ovarian reserve earlier than other hormones such as follicle-stimulating hormone (FSH), luteinizing hormone ( $\mathrm{LH})$, estradiol E2 and other ovarian indicators such as inhibin B and antral follicle count $(\mathrm{AFC})^{6,17-18}$. The aim of our review is to evaluate the impact of surgical technique on the ovarian reserve by comparing the preoperative and postoperative AMH levels of patients who underwent SPL or MPL.

\section{Materials and methods}

\section{Study design and participant}

The present systematic review included all published research comparing the AMH concentration after SPL and MPL for treatment of ovarian benign cysts excluding diseases related to pregnancy. The meta-analysis was performed in accordance with the guidelines for Systematic Reviews and Meta-analyses (PRISMA) based on the authors' predetermined inclusion criteria. Selection of abstracts was conducted by two authors (ET and KT) who independently searched the literature. Only articles written in English were included. Studies considered were randomized clinical trials (RCT), prospective controlled, prospective cohort, or retrospective studies. Institutional Review Board approval was not requested since the present study is a review of published studies.

An electronic database search was performed using Pubmed, Embase and Medline for the identification of studies in English published until September 2019 using a combination of the following search items: singleport laparoscopic cystectomy, ovarian reserve, laparoscopy, ovarian endometrioma, AMH. Other databases were searched and no additional studies were identified. Following the search, all articles considered pertinent on the basis of the title and abstract were retrieved and their reference lists were searched for additional potential studies. Each author independently reviewed the literature. The discrepancies during the data collection were then resolved by consensus of all authors.

Inclusion criteria included age at surgery ( $>18$ years and $<45$ years), BMI between 18,5 and 24,9 (kg/k2), cyst size 3-11 cm, unilateral or bilateral ovarian cysts presumed as benign (endometriomas, dermoid cysts, simple cysts, serous cystadenomas, mucinous cystadenomas) on ultrasonography, regular menstrual cycle (21-35 days). Exclusion criteria were: presence of endocrine disorders, use of any medications such as oral contraceptive pills or other hormonal agents within three months before study enrollment, previous history of ovarian cyst surgery, radiotherapy or chemotherapy and pregnant women. All patients underwent the procedure under general anesthesia with endotracheal intubation and were placed in the Trendelenburg position (Table 1)

\section{Outcome measure}

All studies used the same surgical technique with a few variants and the same biomarker (AMH) to evaluate postoperative ovarian function. Additional evaluation of secondary results included perioperative (operating time, estimated blood loss (EBL), hemoglobin ( $\mathrm{Hb}$ ) drop, adhesiolysis, rupture of cyst, operative complications, conversion rate) and postoperative outcomes (parental analgesic use, postoperative lower abdominal wound pain and length of hospital stay) (Table 2).

\section{Statistical analysis}

Statistical meta-analysis was performed using the RevMan 5.3 software (Copenhagen: The Nordic Cochrane Centre, The Cochrane Collaboration, 2011). Confidence intervals (CI) were set at 95\%. Mean difference (MD) and odds ratios (OR) were used in the analysis. The results were calculated using the DerSimonianLaird random effect model (REM) revealing significant heterogeneity in the methodological characteristics of the included studies ${ }^{19}$. The cut-off for statistical significance was set at $\mathrm{p}<0.05$. Mean values and standard deviations were calculated according to the equations proposed by Hozo et al when not provided by the studies $^{20}$. To assess the between-study heterogeneity more precisely, both the Chi-square based Q statistic test for heterogeneity and the $\mathrm{I}^{2}$ statistic to quantify the proportion of the total variation attributable to heterogeneity were calculated. When there was no statistically significant heterogeneity $\left(\mathrm{p}>0.05, \mathrm{I}^{2}<50 \%\right)$, 
a fixed effect model was adopted. Otherwise, a random effect model was chosen ${ }^{21}$. The heterogeneity was considered statistically significant if a $\mathrm{p}<0.05$. Publication bias was not tested due to heterogeneity of the included studies, which is a confounder that may influence the methodological integrity of these tests.

\section{Results}

Initially the research strategy identified seventeen studies published in the last 10 years. Thirteen articles were excluded on the basis of failure to meet inclusion criteria. Finally the search revealed four relative articles published between 2014 and 2019 and offered 336 patients for this analysis, 143 of which underwent SPL and 193 MPL (Figure 1). Two of the studies were retrospective case-control studies, one was randomized controlled trial and the other was non-randomized concurrent control trial ${ }^{13-16}$. According to the surgical outcomes none of the cases was converted to a different surgical approach and no complication occurred during the perioperative period. Serum AMH was similar between the two groups before the surgery $[\mathrm{MD}=0.04$, $95 \% \mathrm{CI}(0.43,0.52), \mathrm{p}=0.8)$ and after the surgery $[\mathrm{MD}=-0.15,95 \% \mathrm{CI}(0.53,0.23), \mathrm{p}=0.44]$. Thus, the metaanalysis demonstrated similar and non significant reduction in $\mathrm{AMH}$ levels between the two groups four weeks after the procedure irrespective of the surgical technique $[\mathrm{MD}=0.11,95 \% \mathrm{CI}(-0.01,0.24), \mathrm{p}=0.07]$ (Figure 2). Regarding analysis of hospital stay there was also no statistically significant difference between two groups $[\mathrm{MD}=-0.26,95 \% \mathrm{CI}(-0.97,0.45), \mathrm{p}=0.48]$ (Figure 3). Perioperative outcomes analysis revealed that operative time $(\mathrm{min})$ was significantly longer $[\mathrm{MD}=0.57,95 \% \mathrm{CI}(0.30,0.83), \mathrm{p}<0.00001]$ (Figure 4$)$ and blood loss $(\mathrm{ml})$ was significantly higher $[\mathrm{MD}=0.58,95 \% \mathrm{CI}(0.04,1.12) \mathrm{p}=0.03]$ in SPL group (Figure 5). The quality of these results is consistent but poor, as illustrated by the number of the studies, substantial variation in treatment effects and low precision of the point estimates.

\section{Discussion}

Single-port laparoscopic ovarian cystectomy appears to be a promising minimal invasive technique offering the advantages of fast recovery, less trauma, and superior cosmetic outcome. On the other hand, the safety of this technique with respect to aspects of damage to ovarian reserve has been questioned. Until now this relevant issue has been poorly investigated, because of difficulties in assessing ovarian reserve after this kind of surgery in combination with the small number of study subjects and short follow-up intervals.

The presence of an ovarian cyst may distort and damage the adjacent healthy ovarian tissue but the laparoscopic stripping of ovarian cysts may further worsen ovarian reserve, even if performed by experienced surgeons, through at least three ways: (i) the accidental removal of healthy ovarian cortex near the cyst's capsule (ii) the thermal effect of diathermy coagulation of small bleeding vessels on the stripping area with consequent vascular compromise and (iii) a surgery-related local inflammation ${ }^{22-24}$.

In single-port laparoscopy (SPL) surgeons often experience difficulties in handling their instruments due to the unsatisfactory surgical plan. All instruments are placed in parallel, bump each other for targeting procedures and also are in line with the vision ${ }^{25}$. Nevertheless, the default of triangulation could limit the traction and counter traction needed for some ovarian cysts (endometriomas). It is argued that these limitations make the SPL ovarian cystectomy more technically demanding and difficult than conventional MPL and may cause more negative impact on preserving the ovarian function after laparoscopic ovarian cystectomy ${ }^{16}$. Our results have highlighted the fact that while total operative time and blood loss increased using SPL versus MPL ovarian cystectomy, there is not a remarkable difference in hospital stay and in reduction of postoperative AMH between SPL ovarian cystectomy and conventional MPL approach.

In our opinion the development of a new surgical approach should always be compared to the conventional treatment. The majority of studies did not display a remarkable difference in reduction of postoperative AMH between SPL ovarian cystectomy and conventional MPL approach ${ }^{13-15}$.

It seems that besides the number of laparoscopic ports there are several factors like histological type of cyst (endometriotic cysts or non-endometriotic cysts), the hemostatic approach (coagulation, suturing), location of cyst (unilateral or bilateral), cyst size that affect postoperatively the ovarian function ${ }^{15}$. During cystectomy, it is not always easy to identify and separate the cleavage plane between the cyst wall and adjacent ovarian 
cortex tissue due to fibrotic adhesion. Cystectomy using the stripping technique usually leads to removal of normal primary follicles and damage of ovarian reserve. Furthermore, bipolar coagulation at seriously bleeding sites close to ovarian hilus also leads to destruction of the ovarian blood supply and reduced ovarian reserve ${ }^{19}$.

The major limitations of this meta-analysis is that the strength of evidence is low because of quality issues of available studies and the heterogeneity in ovary cyst type of four included studies. Currently the evidence is not strong enough to recommend or discourage the use of single-incision laparoscopy over conventional laparoscopy. The available literature suggests that the risk of complications is similar between procedures. Further randomized studies with larger sample sizes are required to demonstrate in which degree all these factors contribute to the loss of the ovarian reserve.

\section{Variability of AMH}

Several studies have reported that serum AMH levels are mildly decreased in endometriomas than other ovarian tumors and severely declined as the endometriosis is severe and bilateral ${ }^{19}$. Iwase et al reported that decreased AMH levels were found after laparoscopic excision of endometriomas and non-endometriotic cysts but the decrease was more profound in endometriomas than in non-endometriomas ${ }^{26}$. Hirokawa et al also reported that the rate of decline of the serum AMH level was significantly higher in the bilateral group than the unilateral group and was correlated with the revised American Society for Reproductive Medicine( rASRM) score $^{22}$. Laparoscopic cystectomy further exerted a significant negative impact on ovarian reserve measured by serum AMH levels in both short and long term observations ${ }^{27}$. Tsolakidis et al reported that the mean serum AMH level was significantly reduced 6 months after surgery ${ }^{28}$. Chang et al also observed a significant decrease in serum AMH levels 3 months after laparoscopic cystectomy ${ }^{6}$.

\section{Assessment of ovarian reserve}

Ovarian reserve is defined as the functional potential of the ovary, and reflects the number and quality of the follicles left in the ovary at any given time. There are no definite estimates to measure of the quantitative ovarian reserve in reproductive age. Although recent progresses have been achieved in this field, the ideal test reflecting ovarian reserve has remained the evaluation of the response to ovarian stimulation. Various tests and markers of the ovarian reserve have been reported. Hormonal tests such as follicle stimulating hormone (FSH), estradiol, luteinizing hormone (LH), AMH or inhibin-b and sonographic measurements such as ovarian volume, antral follicular count (AFC), or ovarian blood flow have been proposed. Among these factors AMH which is produced by the granulosa cells of the recruited follicles until they become sensitive to FSH, has been identified as a regulator of recruitment for preventing depletion of the entire primordial follicle pool at once $^{29,30}$. Serum AMH levels appears to correspond well with AFCs and ovarian response hyperstimulation in in-vitro ferilization. It has been found to decline with age in females and has been suggested as a predictor of ovarian response and menopausal transition. Moreover, AMH is the only marker of ovarian reserve which is menstrual cycle independent and easily measurable although the threshold levels in follicular and luteal phases need to be standardized. The measurement of serum AMH levels is more convenient than ovarian response to hyperstimulation for predicting the ovarian reserve ${ }^{31}$.

\section{Conclusions}

Based on the outcomes of the present meta-analysis supports the use of SPL as an alternative method to conventional laparoscopy in women with benign ovarian cysts because does not affect the serial change of the serum AMH level. Due to the limited number of the included studies and their primarily retrospective nature the aforementioned outcomes must be interpreted with caution. Further studies with a longer-term follow-up of the ovarian reserve after surgery are needed in the field to clearly evaluate each distinct parameter of the two approaches and to indicate the optimal one for women with fertility problems.

\section{References}

1. Mimoun C, Fritel X, Fauconnier A, Deffieux X, Dumont A, Huchon C. Epidemiology of presumed benign ovarian tumors. J Gynecol Obstet Biol Reprod. 2013; 42:722-729 
2. Kang JH, Kim YS, Lee SH, Kim WY: Comparison of hemostatic sealants on ovarian reserve during laparoscopic ovarian cystectomy. Eur J Obstet Gynecol Reprod Biol. 2015; 194: 64-67

3. Raffi F, Metwally M, Amer S. The impact of excision of ovarian endometrioma on ovarian reserve: a systematic review and meta-analysis. J Clin Endocrinol Metab. 2012; 97: 3146-3154

4. Hong JH, Choi JS, Lee JH, Son CE, Jeon SW, Bae JW. Laparoscopic management of large ovarian tumors: clinical tips for overcoming common concerns. J Obstet Gynaecol. 2012; 38: 9-15

5. Medeiros LR, Rosa DD, Bozzetti MC et al. Laparoscopy versus laparotomy for benign ovarian tumour. Cochrane Database Syst Rev. 2009; 15: CD004751

6. Chang HJ, Han SH, Lee JR et al. Impact of laparoscopic cystectomy on ovarian reserve: serial changes of serum anti-Müllerian hormone. Fertil Steril. 2010; 94: 343-349

7. Iwase A, Hirokawa W, Goto M et al. Serum anti-Müllerian hormone level is a useful marker for evaluating the impact of laparoscopic cystectomy on ovarian reserve. Fertil Steril. 2010; 94: 2846-2849

8. Kitajima M, Khan KN, Hiraki K, Inoue T, Fujishita A, Masuzaki H: Changes in serum anti-Müllerian hormone levels may predict damage to residual normal ovarian tissue after laparoscopic surgery for women with ovarian endometrioma. Fertil Steril. 2011; 95: 2589-2591

9. Celik HG, Dogan E, Okyay E et al. Effect of laparospopic excision of endometriomas on ovarian reserve: serial changes in the serum antimüllerian hormone levels. Fertil Steril. 2012; 97: 1472-1478

10. Mereu L, Angioni S, Melis GB, Mencaglia L. Single access laparoscopy for adnexal pathologies using a novel reusable port and curved instruments. Int J Gynaecol Obstet. 2010; 109: 78-80

11. Jung YW, Choi YM, Chung CK et al. Single port transumbilical laparoscopic surgery for adnexal lesions: a single center experience in Korea. Eur J Obstet Gynecol Reprod Biol. 2011; 155: 221-224

12. Escobar PF, Bedaiwy MA, Fader AN, Falcone T. Laparoendoscopic single-site (LESS) surgery in patients with benign adnexal disease. Fertil Steril. 2010; 93: 2074.e7-10

13. Yoon BS, Kim YS, Seong SJ et al. Impact on ovarian reserve after laparoscopic ovarian cystectomy with reduced port number: a randomized controlled trial. Eur J Obstet Gynecol Reprod Biol. 2014; 176: 34-8

14. Huang BS, Wang PH, Tsai HW, Hsu TF, Yen MS, Chen YJ. Single-port compared with conventional laparoscopic cystectomy for ovarian dermoid cysts. Taiwan J Obstet Gynecol.2014; 53: 523-529

15. Wang D, Liu H, Li D et al. Comparison of the impact of single-port laparoscopic and conventional laparoscopic ovarian cystectomy on the ovarian reserve in adult patients with benign ovarian cysts. Minim Invasive Ther Allied Technol. 2019; 1: 1-8

16. Angioni S, Pontis A, Cela V, Sedda F, Genazzani AD, Nappi. Surgical technique of endometrioma excision impacts on the ovarian reserve. Single-port access laparoscopy versus multiport access laparoscopy: a case control study. Gynecol Endocrinol. 2015; 31: 454-457

17. Alammari R, Lightfoot M, Hur HC. Impact of Cystectomy on Ovarian Reserve: Review of the Literature. J Minim Invasive Gynecol. 2017; 24: 247-257

18. Yuh-Ming Hwu, Frank Shao-Ying Wu, Sheng-Hsiang Li, Fang-Ju Sun, Ming-Huei Lin, Robert KuoKuang Lee. The impact of endometrioma and laparoscopic cystectomy on serum anti-Müllerian hormone levels. Reprod Biol Endocrinol. 2011; 9: 80

19. DerSimonian R, Kacker R. Random-effects model for meta-analysis of clinical trials: an update. Contemp Clin Trial .2007; 28: 105-114

20. Hozo SP, Djulbegovic B, Hozo I. Estimating the mean and variance from the median, range, and the size of a sample. BMC Med Res Methodol. 2005; 5:13 
21. Mantel N, Haenszel W. Statistical Aspects of the Analysis of Data from Retrospective Studies of Disease. J Natl Cancer Inst. 1959; 22: 719-748

22. Hirokawa W, Iwase A, Goto M et al. The post-operative decline in serum anti-Mullerian hormone correlates with the bilaterality and severity of endometriosis. Hum Reprod. 2011;26: 904-910

23. Muzii, L, Bellati, F, Bianchi, A et al. Laparoscopic stripping of endometriomas: a randomized trial on different surgical techniques. Hum Reprod. 2005; 20: 1987-1992 24. Muzii L, Di Tucci C, Di Feliciantonio M et al. Antimüllerian hormone is reduced in the presence of ovarian endometriomas: a systematic review and meta-analysis. Fertility and Sterility. 2018; 110: 932-940.e1

25. Paek J, Nam EJ, Kim YT, Kim SW. Overcoming technical difficulties with single-port access laparoscopic surgery in gynecology: using conventional laparoscopic instruments. Journal of Laparoendoscip and Advanced Surgical Techniques. 2011; 21: 137-141

26. Iwase A, Nakamura T, Nakahara T, Goto M, Kikkawa F. Assessment of ovarian reserve using antiMüllerian hormone levels in benign gynecologic conditions and surgical interventions: a systematic narrative review. Reprod Biol Endocrinol. 2014; 12: 125

27. Hwu YM, Wu FS, Li SH, Sun FJ, Lin MH, Lee LK. The impact of endometrioma and laparoscopic cystectomy on serum anti-Müllerian hormone levels. Reprod Biol Endocrinol. 2011; 9: 80

28. Tsolakidis D, Pados G, Vavilis D et al. The impact on ovarian reserve after laparoscopic ovarian cystectomy versus three-stage management in patients with endometriomas: a prospective randomized study. Fertil Steril. 2010; 94: 71-77

29. Roudebush WE, Kivens WJ, Mattke JM. Biomarkers of Ovarian Reserve. Biomark Insights 2008; 3: 259-268

30. Tremellen KP, Kolo M, Gilmore A, Lekamge DN. Anti-mullerian hormone as a marker of ovarian reserve. Aust N Z J Obstet Gynaecol. 2005; 45: 20-24

31. Jamil Z, Fatima SS,Ahmed K, Malik R. Anti-Mullerian Hormone. Above and Beyond Conventional Ovarian Reserve Markers. Dis Markers. 2016; 2016: 5246217

Table 1. Main characteristics of included studies

\begin{tabular}{llllll}
\hline Year, Author & Patients No & Age(years) & BMI $(\mathrm{Kg} / \mathrm{m} 2)$ & Cyst size, cm & Study ty \\
2014, Yoon & 28 vs 57 & $30,3 \pm 5,0$ vs $30,2 \pm 5,6$ & $20,4 \pm 2,4$ vs $20,7 \pm 2,5$ & $6,1 \pm 1,9$ vs $6,3 \pm 2,1$ & randomi \\
2014, Huang & 34 vs 37 & $34,59 \pm 10,18$ vs $34,73 \pm 9,68$ & $21,29 \pm 2,52$ vs $21,14 \pm 2,61$ & $7,05 \pm 2,37$ vs $6,39 \pm 2,27$ & retrospec \\
2015, Angioni & 49 vs 50 & $30,25 \pm 5,1$ vs $30,53 \pm 4,55$ & $20,4 \pm 2,3$ vs $20,19 \pm 2,58$ & $7,6 \pm 3,56$ vs $7,0 \pm 2,0$ & retrospe \\
2019, Wang & 47 vs 32 & $30,1 \pm 6,8$ vs $29,4 \pm 5,1$ & $20,8 \pm 2,15$ vs $20,65 \pm 1,75$ & $6,5 \pm 1,5$ vs $6,0 \pm 1$ & non-ranc \\
\hline
\end{tabular}

BMI: body mass index, SPL: single-port laparoscopy, MPL: multi-port laparoscopy, AMH: anti-Müllerian hormone

Table 2: Surgical outcomes of included studies

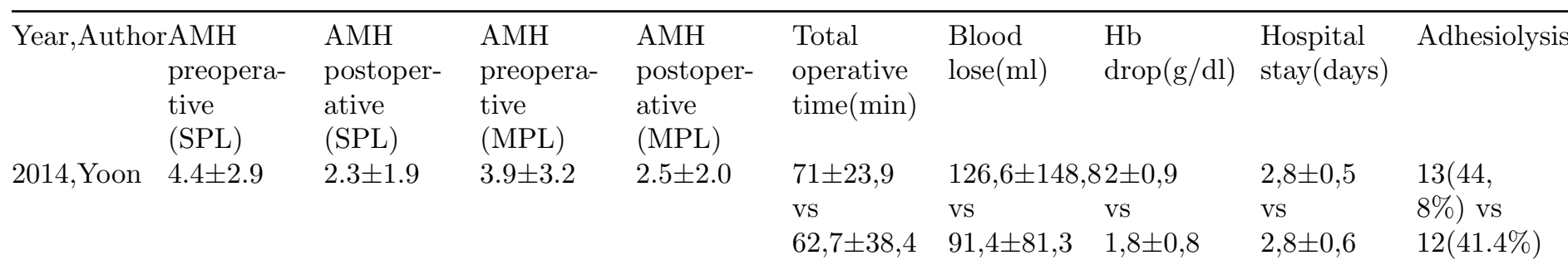




\begin{tabular}{|c|c|c|c|c|c|c|c|c|}
\hline 2014,Huang 3.94 \pm 1.53 & $3.37 \pm 1.24$ & $3.99 \pm 1.75$ & $3.43 \pm 1.52$ & $92,35 \pm 17,1$ & $3108,68 \pm 32$ & $\$ / A$ & $4,03 \pm 1,60$ & $\mathrm{~N} / \mathrm{A}$ \\
\hline & & & & & vs & & & \\
\hline & & & & $83,38 \pm 20,7$ & $98,43 \pm 30,9$ & & $3,78 \pm 0,78$ & \\
\hline 2015 ,Angion2. $90 \pm 2.26$ & $1.76 \pm 1.50$ & $3.13 \pm 2.08$ & $2.09 \pm 1.66$ & $\begin{array}{l}60,3 \pm 15 \\
\mathrm{VS}\end{array}$ & $\begin{array}{l}65 \pm 44,89 \\
\text { vs }\end{array}$ & $\mathrm{N} / \mathrm{A}$ & $\mathrm{N} / \mathrm{A}$ & $\mathrm{N} / \mathrm{A}$ \\
\hline & & & & $45,1 \pm 20,5$ & $28,64 \pm 13,2$ & & & \\
\hline 2019, Wang $3.76 \pm 1.70$ & $3.22 \pm 1.68$ & $3.47 \pm 1.79$ & $3.07 \pm 1.71$ & $50 \pm 10$ & $20 \pm 10$ & $13,1 \pm 7,4$ & $4(4-5)$ & $23(48$ \\
\hline & & & & & vs & & & $9 \%)$ vs \\
\hline & & & & $46 \pm 14$ & $35 \pm 15$ & $15,0 \pm 6,4$ & $5(4-5)$ & $19(59,4 \%)$ \\
\hline
\end{tabular}

AMH: anti-Müllerian hormone, Hb:hemoglobin

Figure 1: PRISMA four-phase flow diagram of search yield, screening and inclusion steps

Figure 2: Forest plot for serum AMH reduction $(\mathrm{ng} / \mathrm{ml})$

Figure 3: Forest plot for hospital stay (days)

Figure 4: Forest plot for operative time (min)

Figure 5: Forest plot for blood loss (ml)

\section{Hosted file}

Table 1!.pdf available at https://authorea.com/users/389850/articles/504380-impact-onovarian-reserve-after-minimally-invasive-single-port-laparoscopic-ovarian-cystectomy-inpatients-with-benign-ovarian-cysts-a-systematic-review-and-meta-analysis

\section{Hosted file}

Table 2!.pdf available at https://authorea.com/users/389850/articles/504380-impact-onovarian-reserve-after-minimally-invasive-single-port-laparoscopic-ovarian-cystectomy-inpatients-with-benign-ovarian-cysts-a-systematic-review-and-meta-analysis

\section{Hosted file}

fig1.pdf available at https://authorea.com/users/389850/articles/504380-impact-on-ovarianreserve-after-minimally-invasive-single-port-laparoscopic-ovarian-cystectomy-inpatients-with-benign-ovarian-cysts-a-systematic-review-and-meta-analysis

\section{Hosted file}

fig2.pdf available at https://authorea.com/users/389850/articles/504380-impact-on-ovarianreserve-after-minimally-invasive-single-port-laparoscopic-ovarian-cystectomy-inpatients-with-benign-ovarian-cysts-a-systematic-review-and-meta-analysis

\section{Hosted file}

fig3.pdf available at https://authorea.com/users/389850/articles/504380-impact-on-ovarianreserve-after-minimally-invasive-single-port-laparoscopic-ovarian-cystectomy-inpatients-with-benign-ovarian-cysts-a-systematic-review-and-meta-analysis

\section{Hosted file}

fig4.pdf available at https://authorea.com/users/389850/articles/504380-impact-on-ovarianreserve-after-minimally-invasive-single-port-laparoscopic-ovarian-cystectomy-inpatients-with-benign-ovarian-cysts-a-systematic-review-and-meta-analysis

\section{Hosted file}


fig5.pdf available at https://authorea.com/users/389850/articles/504380-impact-on-ovarianreserve-after-minimally-invasive-single-port-laparoscopic-ovarian-cystectomy-inpatients-with-benign-ovarian-cysts-a-systematic-review-and-meta-analysis 